\title{
La biodiversité dans les systèmes d'exploitation agricole : le projet IBIS
}

\section{Céline CERVEK}

Chargée de projets environnement-

biodiversité,

Chambre régionale d'agriculture du

Centre,

13, avenue des Droits de I'Homme,

45921 Orleans cedex 9 ,

France

<celine.cervek@centre.chambagri.fr>

\begin{abstract}
The "IBIS" project (acronym for IBIS - to Integrate Blodiversity in farm management Systems) enabled from January 2008 to December 2010 to develop methods and tools to advise on biodiversity at farm scale. This interregional project carried out by the Chambre régionale d'agriculture du Centre (Chamber of Agriculture of the Centre region) was based on a strong collaboration between organisations dealing with farming development, environment, wildlife management, research and education. The project relied on the support of 80 volunteer farmers (cereal producers and cow breeders), which tested the method of farm diagnosis with the help of agricultural technicians and naturalists. This work pointed up the importance for advisors to understand globally how each farm works and the relevance of crossed agricultural and naturalistic competences.
\end{abstract}

Key words: biodiversity, farm, advice, diagnosis
La prise en compte de la biodiversité sur les exploitations agricoles est une préoccupation récente et complexe, qui mobilise des compétences variées, allant de l'agronomie à l'écologie, en passant par la compréhension du fonctionnement des systèmes d'exploitations agricoles. La biodiversité est une vaste thématique, difficile à appréhender, dont la connaissance n'est d'ailleurs que très partielle. Les agents du développement agricoles manquent souvent d'expérience et de références sur ce sujet pour pouvoir répondre aux questions très concrètes des agriculteurs. Pourtant, la biodiversité devient de plus en plus présente dans les politiques publiques, du fait du constat de son érosion mais aussi en lien avec une prise de conscience des intérêts qu'elle remplit en termes de fonctions et de services, pour la société et pour l'agriculture.

Le projet IBIS avait pour objectifs de rassembler des références existantes, d'élaborer et de tester des méthodes de diagnostic et de conseil co-construit avec l'agriculteur. II s'agissait d'apporter des outils aux agents du développement agricole pour les aider à accompagner les agriculteurs sur cette thématique (figure 1).

Après une présentation du projet, des outils produits et de la démarche de diagnostic-conseil menée sur les exploitations, cet article livre les principaux enseignements tirés des retours d'expériences des conseillers et des agriculteurs.

\section{Le projet}

Le projet Intégrer la biodiversité dans les systèmes d'exploitations agricoles (IBIS) a été ainsi nommé car son objectif de départ était de tenter de comprendre comment la prise en compte de la biodiversité peut être améliorée, à l'échelle de l'exploitation agricole, en cohérence avec le fonctionnement global du système d'exploitation, les objectifs de l'agriculteur et les enjeux présents au niveau du territoire. Ce projet a été déposé par la Chambre régionale d'agri- culture du Centre puis sélectionné dans le cadre de l'appel à projets de développement agricole et rural de 2007 (financement CasDAR du ministère de l'Agriculture). Il s'est déroulé de janvier 2008 à décembre 2010 et a associé un large partenariat issu de 16 départements et quatre régions (Centre, Lorraine, Picardie, Poitou-Charente) (figure 2): chambres d'agriculture, fédérations de chasseurs, Conservatoires d'espaces naturels, Office national de la chasse et de la faune sauvage (ONCFS), associations naturalistes, recherche et enseignement, instituts techniques. . (encadré 1).

Le projet IBIS avait pour finalité de rassembler des références sur la biodiversité en lien avec les pratiques agricoles et le fonctionnement des exploitations pour :

- améliorer la prise en compte de la biodiversité dans le conseil à l'échelle de l'exploitation agricole ;

- créer des outils pour les acteurs du développement agricole et formaliser une démarche de diagnostic-conseil ;

Pour citer cet article : Cervek C. La biodiversité dans les systèmes d'exploitation agricole : le projet IBIS. OCL 2011 ; $18(3)$ : $158-67$. doi : 10.1684/ocl.2011.0382 


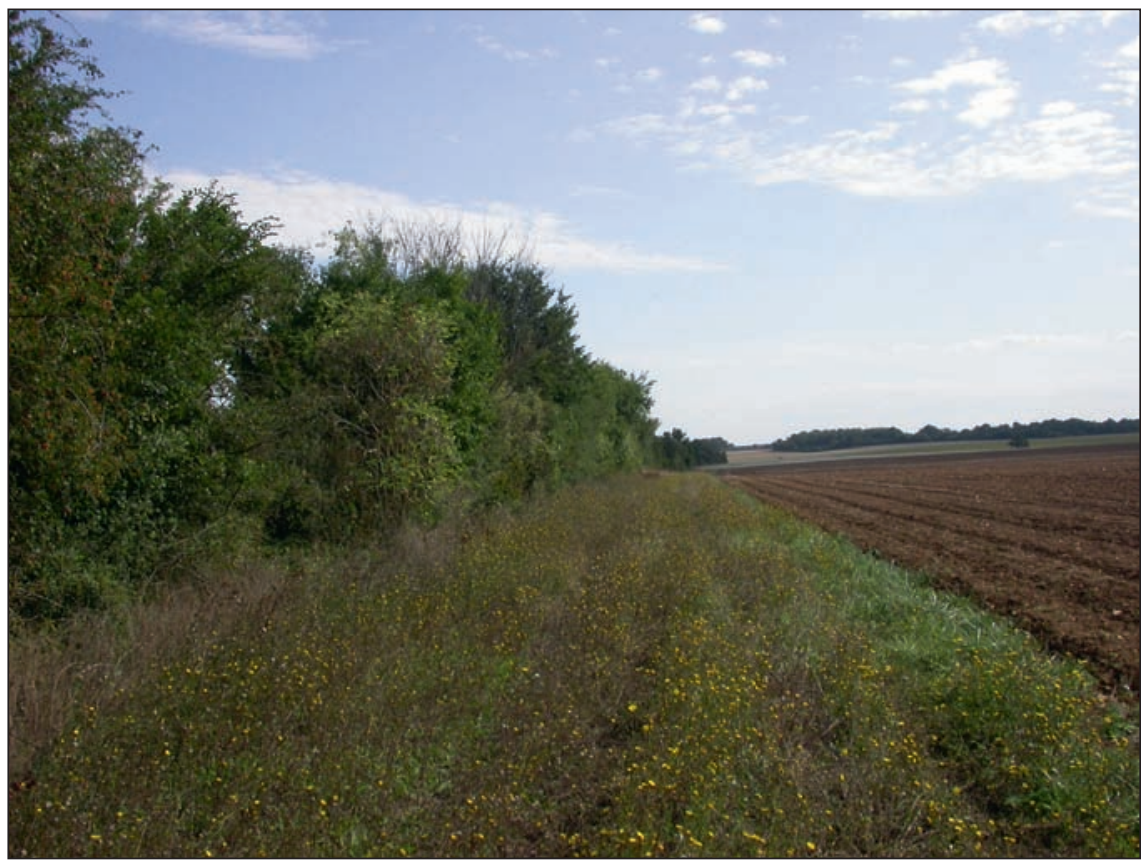

Figure 1. Exemple d'aménagement particulierement favorable à la biodiversité : haie composée d'essences locales bordée d'une bande enherbée pérenne spontanée, située entre 2 parcelles, en zone de plaine céréalière ouverte.

- renforcer le lien entre recherche, développement et formation sur cette thématique, faciliter le transfert des connaissances et renvoyer des questions vers la recherche.

Les travaux menés dans le cadre du projet ont porté sur les systèmes de production en grandes cultures et en polyculture élevage bovin, situés sur des milieux allant de la grande plaine ouverte au bocage en passant par des milieux intermédiaires.

Le projet comprenait trois grands volets :

- la constitution d'un référentiel sur les aménagements et pratiques favorables à la biodiversité, à destination des conseillers ;

\section{Encadré 1 \\ Le partenariat}

Le projet Intégrer la biodiversité dans les systèmes d'exploitation agricoles (IBIS), piloté par la Chambre régionale d'agriculture du Centre a associé des acteurs de tous horizons répartis dans 16 départements (figure 2):

les Chambres d'agriculture et les Fédérations de chasseurs du Centre, de Picardie, de Lorraine, des Deux-Sèvres, de Charente-Maritime et du Maine et Loire ; I'Office national de la chasse et de la faune sauvage ; les Conservatoires régionaux d'espaces naturels de Poitou-Charentes, Picardie et Lorraine ; la Ligue pour la protection des oiseaux de Touraine, d'Anjou et de Charente-Maritime ; I'Association Hommes et Territoires; les Services régionaux de la formation et du développement des DRAAF et les lycées agricoles de Bretagne et du Centre, Agrocampus Ouest site de Beg Meil ; le Centre d'Expertise et de Transfert Universitaire Innophyt de I'Université de Tours; le Centre régional de la propriété forestière de Picardie, I'INRA SAD Paysage de Rennes, I'INRA de Nancy, I'INRA SAD de Mirecourt, AgroParisTech, l'École supérieur d'agriculture d'Angers, Arvalis-Institut du végétal, la Fédération nationale des chasseurs, le CEZ-Bergerie Nationale de Rambouillet, l'Assemblée permanente des Chambres d'Agriculture.

Le projet IBIS a bénéficié du soutien financier du ministère de I'Alimentation, de l'Agriculture et de la Pêche au travers du fonds Casdar (Compte d'affectation spécial pour le développement agricole et rural).

- l'élaboration d'une démarche de diagnostic-conseil et le test sur 80 exploitations agricoles ;

- I'analyse des résultats, la formalisation et la diffusion d'une boîte à outils.

\section{Constitution d'un référentiel des pratiques agricoles en lien avec la biodiversité}

Ce volet visait à capitaliser les références existantes, pour élaborer un référentiel sur les aménagements et pratiques agricoles favorables à la biodiversité. II a permis de construire un outil d'aide au conseil, composé de fiches techniques sur les pratiques et aménagements qui peuvent être préconisés selon le contexte. Ce référentiel renseigne à la fois sur les modalités de mise en œuvre de ces pratiques mais également sur leurs avantages et contraintes, sur les plans environnemental, agronomique, socio-économique et réglementaire.

Ce travail de recherche bibliographique et de rédaction, qui s'est appuyé sur de la bibliographie scientifique et de la " littérature grise ", a été réalisé et validé collectivement par un groupe de travail composé de 16 rédacteurs issus des différents types d'organismes associés au projet. Ces fiches ont été relues puis améliorées en partenariat étroit avec le comité scientifique IBIS et avec 54 relecteurs qualifiés (recherche, naturaliste, enseignement et développement).

Vinqt-quatre fiches-conseil ${ }^{1}$ ont été ainsi produites (tableau 1), ainsi qu'un "guide introductif", document transversal pour un raisonnement global à l'échelle de l'exploitation et du territoire, expliquant le contexte et les enjeux de la prise en compte de la biodiversité.

On notera néanmoins que des difficultés d'accès et d'utilisation des références de la recherche ont été rencontrées. En effet, il existe une multitude de publications, souvent en anglais, qui abordent la biodiversité chacune sous des angles très spécifiques et précis et non généralisables, d'où la difficulté pour les organismes de développement à

\footnotetext{
${ }^{1}$ L'ensemble de ces fiches et leurs annexes sont téléchargeables sur internet: http:// www.centre.chambagri.fr/developpementagricole/ibis.html
} 
Tableau 1. Liste des fiches du référentiel sur les pratiques et aménagements favorables à la biodiversité.

\begin{tabular}{|ll|}
\hline Aménagements & Pratiques \\
\hline Bandes enherbées & Travail du sol \\
\hline Couverts faunistiques et floristiques & Cultures intermédiaires \\
\hline Couverts pollinisateurs & Raisonnement des produits phytosanitaires \\
\hline Couverts fleuris & Méthodes alternatives aux phytosanitaires \\
\hline Bordures de champs & $\begin{array}{l}\text { Absence de traitement en bordure } \\
\text { de parcelle de céréales }\end{array}$ \\
\hline Arbres isolés & Gestion de l'irrigation \\
\hline Haies & Travaux de récolte (hors prairie) \\
\hline Bosquets, boqueteaux, buissons & Prairies \\
\hline Ripisylves & Prés-vergers \\
\hline $\begin{array}{l}\text { Fossés de drainages } \\
\text { et autres fossés }\end{array}$ & Agroforesterie \\
\hline Mares et mouillères & Miscanthus et switchgrassn \\
\hline Bâti agricole & Taillis à courte et très courte rotation \\
\hline
\end{tabular}

s'approprier et vulgariser les résultats de la recherche. De plus, ces résultats sont encore très parcellaires et partiels. Des synthèses comme celle de I'ESCo ont néanmoins facilité les recherches bibliographiques du groupe de travail, mais celui-ci s'est en grande partie basé sur des résultats de littérature grise, du fait de leur caractère plus appliqué.

\section{Élaboration d'une démarche de diagnostic- conseil d'exploitation sur la biodiversité, en s'appuyant sur un réseau de 80 fermes}

Deux groupes de travail ont été chargés, avec l'appui d'un comité scientifique, de définir la méthodologie et d'organiser les tests :

- "GtC » composé d'agents de chambres d'agriculture, conservatoires d'espaces naturels, fédérations de chasseurs, ONCFS, instituts techniques, enseignement agricole, chercheurs ;

- et "GtNaturaliste ", plus spécifiquement chargé de formaliser la partie naturaliste des diagnostics (études des milieux, faune et flore...), composé d'agents à compétences naturalistes issus des différentes structures partenaires (associations naturalistes, conservatoires d'espaces naturels, fédérations de chasseurs, ONCFS, chambres d'agriculture...).
Ce volet visait à bâtir une méthode de diagnostic prenant en compte à la fois I'approche globale d'exploitation et la composante plus naturaliste de la biodiversité

Le travail s'est organisé en trois phases :

- définitiond'uneméthode de diagnosticconseil d'exploitation ;

- test du diagnostic sur 80 exploitations agricoles, par binômes de conseillers ;

- recueil des retours d'expériences des agriculteurs et des conseillers pour en tirer les enseignements et améliorer la méthode et les outils.

\section{Définition de la méthode de diagnostic-conseil}

La définition de la méthode de diagnostic-conseil a été réalisée à partir des réflexions de deux groupes (GtC et GtNaturaliste) de travail interrégionaux. La première étape a été d'inventorier les méthodes existantes de diagnostic. Trois principaux types de méthodes ont été recensés :

- des diagnostics agri-environnementaux, composés généralement d'indicateurs, avec barèmes ou note, avec un volet biodiversité plus ou moins développé ;

- des méthodes " naturalistes ", de type inventaires/suivis d'espèces, nécessitant généralement des compétences en termes d'identifications d'espèces/milieux ainsi que du temps important pour la réalisation ;

- des méthodes avec questions plus ouvertes, visant à comprendre le système d'exploitation agricole.

Dans le cadre du projet IBIS, la méthode qui a été construite par les groupes de travail s'est orientée vers une méthode " ouverte ", non uniquement basée sur des indicateurs, que l'on peut qualifier "d'approche globale de l'exploitation, des milieux et des pratiques agricoles, appliquée à la biodiversité ".

En effet, ce type de méthode a été retenu afin de privilégier la compréhension et l'échange entre les conseillers et l'agriculteur. Nous sommes partis du postulat que, avant d'apporter un conseil, il fallait déjà comprendre le fonctionnement de l'exploitation agricole : afin de prendre en compte les contraintes de l'agriculteurs, ses projets, ses objectifs...

La difficulté induite : ce diagnostic fait appel à de multiples compétences de la part du conseiller étant donné que la méthode ne lui apporte pas de conclusions toutes faites mais qu'il doit se forger sa propre analyse à partir de références et de connaissances qui sont mises à sa dispositions par la méthode.

La figure 3 donne le déroulement type d'un diagnostic.

\section{La réalisation des tests du diagnostic}

Dans le cadre du projet IBIS, les conseillers qui ont réalisé les diagnostics tests ont reçu préalablement une formation de deux jours à la méthode, comprenant des explications théoriques et un test, en groupe (réalisé par région) sur une exploitation agricole.

Les 80 agriculteurs volontaires pour tester la méthode (cinq par département) ont été choisis de façon à avoir une représentation de différentes situations dans les systèmes étudiés, en respectant les proportions suivantes:

- $50 \%$ des exploitations en grandes cultures, $50 \%$ en polyculture élevage bovin ;

- représentation des milieux ouverts à fermés, en plaine ;

- la moitié des agriculteurs déjà sensible à la thématique, avec différentes motivations possibles (environnementale, cynégétique, agronomique...), l'autre 


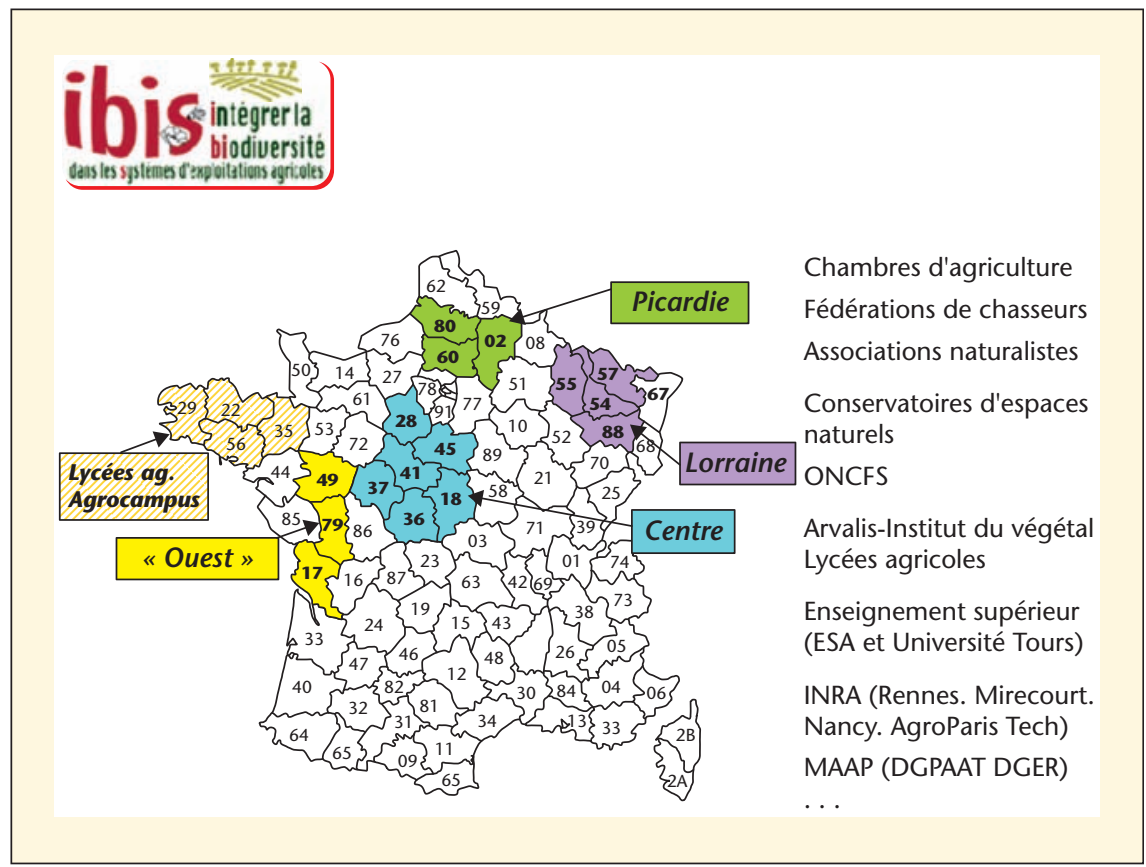

Figure 2. Zones géographiques et types de partenaires impliqués dans le projet IBIS.

moitié non sensible à la thématique ( $n^{\prime}$ ayant pas mis en place d'actions avec un objectif lié à la biodiversité et non particulièrement intéressé par le sujet).

La réalisation des 80 diagnostics tests a mobilisé une cinquantaine d'agents, qui sont intervenus en binômes ou trinômes aux compétences complémentaires, associant à chaque fois un conseiller agricole et un conseiller à compétences naturalistes (issu, selon les départements, d'une fédération des chasseurs d'un conservatoire d'espaces naturels, d'une association naturaliste ou de I'ONCFS...), afin de permettre des échanges de connaissances et de savoir-faire. En effet, très rares sont les agents qui peuvent prétendre avoir une connaissance suffisante pour aborder à

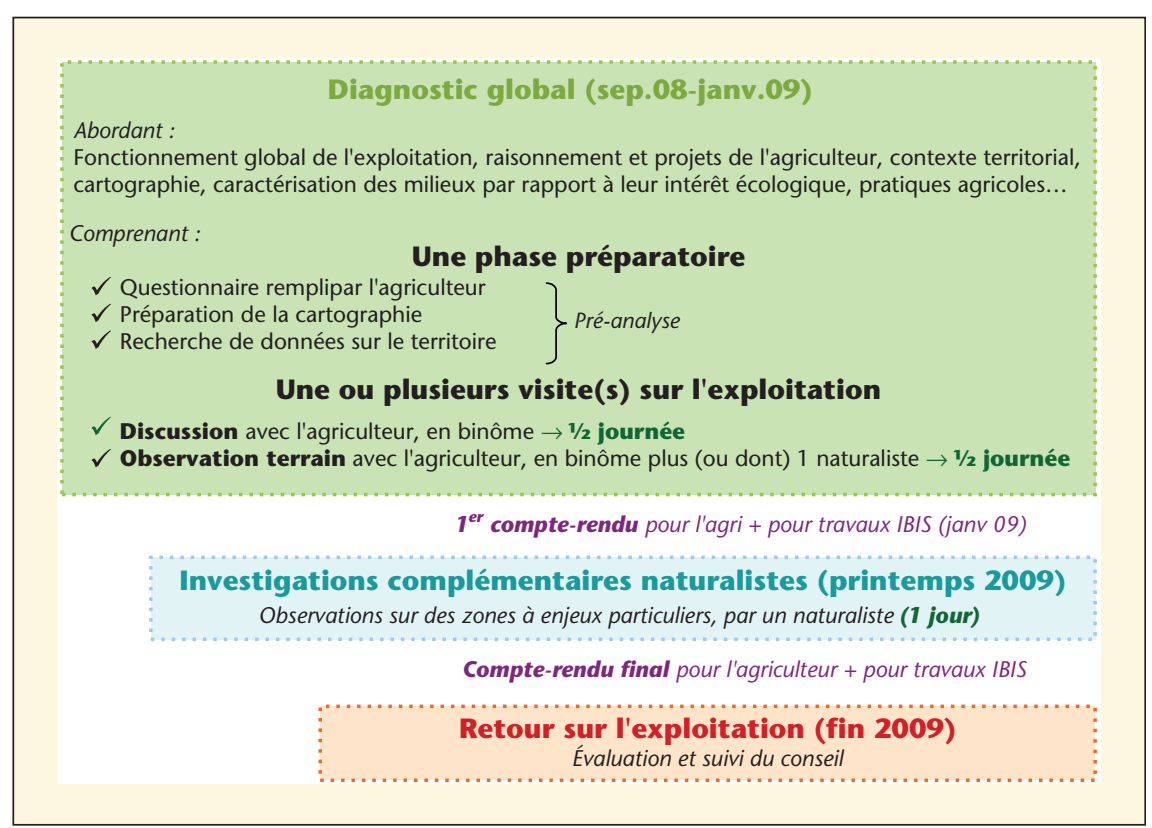

Figure 3. Déroulement type d'un diagnostic. la fois l'approche globale d'exploitation et les composantes plus naturalistes.

La méthode a également été testée avec des étudiants de lycées agricoles, en Bretagne, dans un cadre pédagogique mené par les équipes enseignantes.

Les tests se sont déroulés en trois phases.

\section{Automne-hiver 2008 :}

\section{phase de diagnostic global}

La phase de diagnostic global est, composée d'une phase préparatoire et d'une ou plusieurs visites des conseillers sur l'exploitation, pour un échange avec l'agriculteur.

Lors de la phase préparatoire, I'agriculteur remplit un questionnaire (environ une demi-heure) qui renseigne sur ses productions, ses pratiques, ses projets, ses motivations, la vision qu'il a du territoire, ses objectifs par rapport à la biodiversité. Les conseillers quant à eux préparent les données qu'ils ont sur le territoire (zonages environnementaux, données connues d'inventaires, milieux, espèces potentiellement présentes, cartographies...) et récupèrent les cartes de l'exploitation (contour des parcelles de l'exploitation sur orthophotoplan et fond de carte IGN).

L'analyse du questionnaire préalable complété par l'agriculteur et des données sur le territoire permet aux conseillers d'avoir une vue d'ensemble de l'exploitation et du territoire, et de prévoir les types d'enjeux qui pourront être rencontrés sur l'exploitation.

Les visites sur l'exploitation permettent $d^{\prime}$ affiner cette analyse au travers d'un échange avec l'agriculteur, sur ses pratiques, ses projets. . La cartographie (assolement et éléments fixes du paysage), renseignée avec lui est un bon support pour visualiser la situation initiale et commencer à esquisser des pistes d'aménagements. La visite terrain avec l'agriculteur permet d'observer les zones à enjeux et de discuter des aménagements possibles.

\section{Printemps 2009 : compléments d'observations naturalistes}

Les investigations naturalistes complémentaires ont avant tout eu un rôle pédagogique et non un rôle d'évaluation de l'état de la biodiversité. Il s'agissait de montrer à l'agriculteur la biodiversité présente sur son exploitation pour lui apporter un nouveau regard sur son territoire, lui expliquer l'écologie des espèces, le lien avec les milieux présents 
et ses pratiques, les intérêts de préserver telle ou telle espèce... Dans le cadre du projet nous aurions souhaité standardiser un protocole commun pour l'ensemble des fermes, mais cela s'est avéré difficile. En effet, le faible temps d'observation imparti pour cette étape (de l'ordre d'une journée) ne permettait pas de réaliser des inventaires complets. De plus, les milieux et les espèces qui peuvent être observés dépendent naturellement du territoire, de la période de passage ainsi que des connaissances de l'expert naturaliste. Dans le cadre du projet IBIS, cette phase a donc pris différentes formes, comme: la réalisation de relevés floristiques (quadrats), de points d'écoutes oiseaux ou encore de " promenade naturaliste » sur l'exploitation afin d'observer avec un expert naturaliste les espèces visibles à l'instant de la visite.

\section{Les comptes-rendus}

Les diagnostics-tests ont donné lieu à la rédaction d'un compte-rendu formalisé et détaillé, pour l'agriculteur, selon une trame commune, comprenant notamment une cartographie de l'exploitation sous SIG et des propositions d'améliorations à mettre en œuvre. Les différents outils formalisés dans le cadre du projet et notamment le référentiel des pratiques et aménagements favorables à la biodiversité ont pour but d'aider les conseillers dans leurs préconisations.

\section{Recueil des retours d'expériences des agriculteurs et des conseillers pour en tirer les enseignements et améliorer la méthode et les outils}

Suite à chaque phase de test, des réunions de bilan ont eu lieu, dans chacune des quatre régions concernées, afin de capitaliser les retours d'expériences des agents de terrain. Le bilan général de ces réunions a permis de lancer le travail d'amélioration des outils et de la méthode de diagnostic-conseil. Cette analyse a également permis d'appréhender le raisonnement des agriculteurs au regard de la biodiversité et d'évaluer différents modes de conseil. Les avis des agriculteurs ont été recueillis après la réalisation des diagnostics, au travers d'un questionnaire d'évaluation de la démarche ( $c f$. paragraphe suivant).

Ces diagnostics-tests et leur analyse ont permis de produire une méthodologie reproductible, sous forme d'un guide accompagné d'outils d'appui (questionnaire, clé d'interprétation, fiche de caractérisation des milieux...).

\section{Analyse des résultats, formalisation et diffusion d'une boîte à outils}

L'année 2010, dernière année du projet, a été consacrée à l'analyse, la formalisation et à la diffusion des résultats.

\section{Les outils produits}

Le projet IBIS a abouti à la construction d'une boîte à outils à destination des conseillers, comprenant :

- un référentiel sur les pratiques et aménagements favorables à la biodiversité, composé de 24 fiches techniques d'aide au conseil, présentant les intérêts des mesures pouvant être préconisées, apportant des recommandations techniques, des éléments de chiffrage des coûts... Exemples de fiches: bandes enherbées, couverts pollinisations, haies, mares, travail du sol, cultures intermédiaires... ;

- Une méthode de diagnostic-conseil d'exploitation sur la biodiversité, basée sur une analyse globale du fonctionnement de l'exploitation agricole, des enjeux du territoire, des milieux présents et des pratiques agricoles, ainsi que sur des observations complémentaires naturalistes. II permet de coconstruire avec l'agriculteur un plan d'action ;

- divers éléments : guide introductif expliquant le contexte et les enjeux, argumentaire pour la prise en compte de la biodiversité en milieu agricole, témoignages d'agriculteurs, exemples de comptes-rendus de diagnostics rédigés, fiches pédagogiques d'appui au diagnostic sur les espèces et les milieux...

Le colloque national de restitution du projet IBIS a eu lieu le 16 novembre 2010 à Paris. II a rassemblé près de 200 participants, avec des retours très positifs. La "boîte à outils " a été distribuée sous forme d'un CD-rom.

L'ensemble des outils produits est aujourd'hui disponible sur Internet, sur le site : http://www.centre.chambagri. $\mathrm{fr} /$ developpement-agricole/ibis.html
L'analyse de la démarche menée et les principaux enseignements

Une élève ingénieur a réalisé son stage de fin d'études sur l'analyse des éléments contenus dans les dossiers des 80 exploitations : données des questionnaires préalables complétés par les agriculteurs (renseignant notamment sur leurs pratiques en place, leur perception de la biodiversité, leurs motivations...), comptes-rendus des diagnostics (préconisations, cartographie...), fiches d'évaluation de la démarche par les agriculteurs (58 ont été retournées), retours d'expériences des conseillers...

Les enseignements qui en ressortent sont les suivants.

\section{Les principaux avis formulés par les conseillers}

Globalement, les retours des conseillers sont positifs sur la méthode de diagnostic, qui permet d'aborder la biodiversité au travers d'une approche globale du fonctionnement de l'exploitation resituée dans son milieu et prenant en compte les objectifs et la stratégie de l'agriculteur. La principale difficulté soulevée est le temps nécessaire pour la réalisation d'un diagnostic (de l'ordre de trois jours, du premier contact à la remise du compte-rendu) qui rend son coût élevé, hors d'un cadre expérimental, même si l'on peut espérer qu'en phase de routine et après une bonne appropriation de la méthode, les conseillers pourront optimiser ce temps. Néanmoins la plupart des conseillers s'accordent à dire que ce temps est nécessaire pour faire un diagnostic complet et de qualité.

La complémentarité entre les conseillers, au sein des binômes ou trinômes a été jugée, à l'unanimité, très enrichissante : les naturalistes disent avoir progressé dans la connaissance du fonctionnement de l'exploitation permettant des conseils plus pertinents; les conseillers agricoles disent avoir acquis des connaissances sur la biologie des espèces permettant d'argumenter des préconisations sur les pratiques et aménagements.

La liaison entre approche globale de l'exploitation et l'approche naturaliste a donc été des plus riches.

Au cours des échanges avec les agriculteurs, différents freins et leviers à la prise en compte de la biodiversité ont pu être identifiés. La figure 4 en donne une liste non exhaustive. 


\begin{tabular}{|l|l|}
\hline \multicolumn{2}{|c|}{ Facteurs bloquant la mise en place d'actions par les agriculteurs } \\
\hline Administratif & $\begin{array}{l}\text { Réglementation : crainte des contrôles (conditionnalité) } \\
\text { Contraintes administratives supplémentaires (MAE) } \\
\text { Ne pas être propriétaire }\end{array}$ \\
\hline $\begin{array}{l}\text { Technico- } \\
\text { économique }\end{array}$ & $\begin{array}{l}\text { Coût des aménagement/changements de pratiques } \\
\text { Temps disponible, contraintes dans l'organisation du travail } \\
\text { Incidences techniques/agronomiques, sécurisation du système } \\
\text { Trouver des débouchés pour la production (avant de diversifier assolement) } \\
\text { Achat de nouveau matériel, nouvelle technique à acquérir }\end{array}$ \\
\hline $\begin{array}{l}\text { Manque } \\
\text { d'information }\end{array}$ & $\begin{array}{l}\text { Méconnaissance de la biodiversité, manque d'information sur ce qui est réalisable } \\
\text { Ne voient pas l'intérêt pour leur exploitation }\end{array}$ \\
\hline Image & $\begin{array}{l}\text { Regard des autres agriculteurs, ne veulent pas être tout seuls à faire la démarche } \\
\text { Manque de reconnaissance de la société }\end{array}$ \\
\hline Psychologique & $\begin{array}{l}\text { Peur du changement } \\
\text { Réticence psychologique par rapport à la représentation de la « biodiversité » }\end{array}$ \\
\hline Territorial & Parcellaire dispersé \\
\hline
\end{tabular}

Les principaux avis formulés par les agriculteurs sur la démarche dans les questionnaires d'évaluation (58 réponses) sont les suivants :

- une satisfaction, de la part de $75 \%$ des agriculteurs participants, par rapport aux diagnostics-conseils réalisés, $85 \%$ sont prêts à recommander le diagnostic IBIS à leurs collègues;

- les causes d'insatisfaction (pour les $25 \%$ non satisfaits): le manque de connaissance ou d'implication de la part du conseiller, l'absence de proposition ou des préconisations non réalisables (raisons techniques, économiques ou en désaccord avec les objectifs de I'agriculteur);

- ont été particulièrement appréciés :

- un regard extérieur sur leur exploitation, d'autant plus que la biodiversité est un thème qu'ils ont peu I'habitude d'aborder avec leurs conseillers habituels,

- I'apport de connaissances nouvelles, notamment la remise de document pédagogiques,

- I'évaluation de leurs pratiques (points positifs et négatifs),

- I'aide à la construction de projet,

- la cartographie de l'exploitation (avant/après aménagement, qui permet de bien visualiser les projets).

Figure 4. Principaux freins et leviers à la mise en place d'actions en faveur de la biodiversité par les agriculteurs.

\section{Les principaux avis formulés par les agriculteurs}

Remarque préalable : notre échantillon de 80 agriculteurs n'était pas représentatif de l'ensemble des agriculteurs. En effet, malgré les critères de choix des exploitations fixés initialement, il s'est avéré que notre panel d'agriculteurs était déjà sensible à l'environnement: en témoigne l'analyse des questionnaires préalable qui montre une plus forte proportion, comparée à la moyenne nationale, d'agriculteurs engagés dans des démarches contractuelles ou de qualification en faveur de l'environnement.

Pour autant la biodiversité n'est pas une finalité en soi pour la plupart d'entre eux. En effet, à la question " quels sont vos objectifs ", à choisir dans une liste de dix possibilités (figure 5), les premières réponses mentionnées sont " maintenir le potentiel agronomique du sol » et " limiter les intrants ". Cela atteste que les finalités agro-économiques priment. Donner une entrée agronomique au conseil sur la prise en compte de la biodiversité et démontrer ses intérêts sur ce plan, semble donc être un nécessaire levier pour sensibiliser une majorité d'agriculteurs.

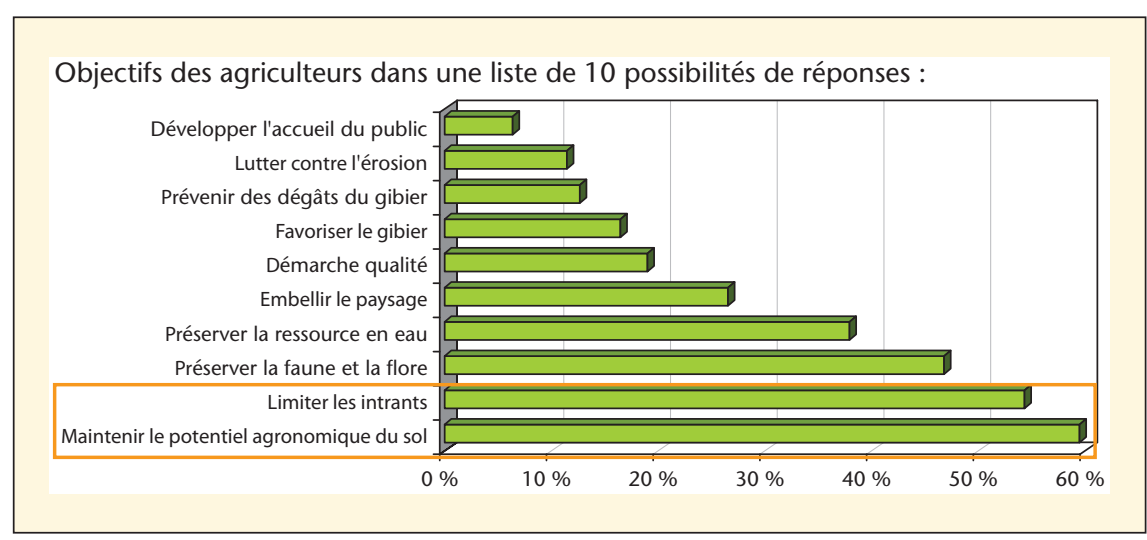

Figure 5. Résultats des réponses des 80 agriculteurs participants, à une question extraite du questionnaire préalable au diagnostic.
Les attentes complémentaires formulées par les agriculteurs :

- améliorer la précision du conseil apporté (détails techniques et réglementaires) exemple : sur la nature des couverts à implanter, où se procurer les semences, le coût, etc ;

- aller plus loin en termes d'acquisition de références locales, d'échanges tech- 
Tableau 2. Principaux types de préconisations figurant dans les comptes-rendus des 80 diagnosticstests (l'effectif correspond au nombre de fois où la préconisation a été formulée).

\begin{tabular}{|ll|}
\hline Préconisation & Effectif \\
\hline Entretien d'éléments fixes du paysage (haies, bosquets. . .) & 38 \\
\hline Plantation d'éléments fixes du paysage & 33 \\
\hline Plantation et/ou entretien de bandes enherbées & 25 \\
\hline Mise en place ou entretien de « jachères » (JEFS, mellifères, fleuries. . .) & 12 \\
\hline Autres création ou entretien d'éléments fixes (mares, ripisylves, fossés) & 10 \\
\hline Date de broyage des jachères ou sens de fauche des prairies & 26 \\
\hline Mise en place ou entretien de couverts d'intercultures & 23 \\
\hline $\begin{array}{l}\text { Diversification de l'assolement, allongement de rotation, } \\
\text { taille des parcelles }\end{array}$ & 22 \\
\hline Gestion des prairies (fertilisation, chargement. ..) & 8 \\
\hline $\begin{array}{l}\text { Réduction des produits phytosanitaires (sur les bords de champs, } \\
\text { les éléments fixes du paysage et les parcelles) }\end{array}$ & 10 \\
\hline Travail du sol simplifié & 10 \\
\hline Gestion des cultures (dates de semis, matériel. . .) & 4 \\
\hline
\end{tabular}

niques avec d'autres agriculteurs, d'indicateurs pour se situer ou de suivis faune-flore.

Pour deux cinquièmes des agriculteurs ayant répondu au questionnaire d'évaluation, le diagnostic est le déclencheur à la mise en place d'actions envisagées. À noter qu'aucun financement n'était possible, dans le cadre du projet IBIS, pour ce type d'actions.

L'analyse des comptes-rendus des diagnostics renseigne également sur les préconisations qui ont été faites, comme le montre le tableau 2.

On peut ainsi remarquer que la majorité des préconisations qui ont été faites concernent l'implantation ou l'entretien des éléments fixes du paysage.

\section{Conclusions}

Le projet IBIS a permis de développer des méthodes et outils pour le conseil sur la biodiversité à l'échelle de l'exploitation agricole à destination des agents du développement agricole: guide introductif de présentation des enjeux, référentiel sur les pratiques favorables à la biodiversité, méthode de diagnosticconseil d'exploitation, fiches sur les milieux et espèces, témoignages d'agriculteurs, argumentaire...

Les outils IBIS ont fait I'objet d'un consensus entre différents acteurs. Ils sont maintenant entre les mains des organismes qui souhaitent se les approprier.

\section{POUR EN SAVOIR PLUS}

AFAHC. Guide technique PAGESA: guide d'aménagement et de gestion des systèmes

Agence de I'Eau-Rhin-Meuse. Guide de gestion de la végétation des bords de cours d'eau. Publication AERM, 2000.

Agence de I'Eau-Rhin-Meuse, Chambres d'agriculture d'Alsace-Lorraine, MEDAD, DDAF Lorraine. Les ripisylves: des systèmes compatibles avec votre activité agricole. Publication AERM, 2008.

Arnold N, Ovenden D. Le guide herpéto. Les guides du naturaliste. Delachaux et Niestlé, $2004: 288$ p.

APCA. L'agroforesterie dans les réglementations agricoles, état des lieux en juin. 2010.

Archambeaud M. Intensifier la production agricole grâce à l'agroforesterie. 2008.

Arnabo Idi F, Alban N. La gestion des mares de plaine/Guide technique. Édition Office national des forêts, 2007: 207 p.

Arthur L, Lemaire M. Les Chauves-souris de France, Belgique, Luxembourg et Suisse. Paris : Biotope, Mèze (Collection Parthénope) : Muséum national d'histoire naturel, 2009 : $544 \mathrm{p}$.

Aubertot JN, Clerjeau M, David C, et al. Expertise scientifique collective "Pesticides, agriculture et environnement ". France : INRA et CEMAGREF, 2005.

Baudry O, Bourgery C, Guyot G, Rieux R. Les haies composites réservoirs d'auxiliaires. Paris : Centre technique interprofessionnel des fruits et légumes/Hortipratic, 2000. Organisation, dynamique et gestion. Paris : Éditions INRA, 2003. agroforestiers. 2009.

Baudry J, Jouin A. De la haie aux bocages -
Bernard JL, Havet P, Fort M. Productions végétales, pratiques agricoles et faune sauvage. Éditions UIPP, ACTA, ONCFS, 2008.

Bertrand J. Agriculture et biodiversité, un partenariat à valoriser. Educagri éditions, ONCFS, 2001.

Bersonnet C, Froissard D, Lemesle B. Impact écologique de l'insertion de Miscanthus giganteus et de Switchgrass Panicum virgatum en Indre-et-Loire à travers les populations de carabes, 2008.

Bourdeaux A. Élaboration d'un référentiel des pratiques agricoles favorables à la biodiversité. Thèse : Sciences agronomiques. École nationale d'agriculture d'Angers, $2007: 60 \mathrm{p}$.

Bournerias M, Arnal G, Bock C. I. Guide des groupements végétaux de la région parisienne. Paris : Belin, 2001 : 640 p.

Bro E. La faune sauvage en milieux cultivés. ONCFS, 2007.

Bro E, Joannon A, Thenail C, Baudry J, Mayot P. Aménagement de I'habitat pour la Perdrix grise en plaine de grande culture. À la recherche de compromis avec les agriculteurs. Faune Sauvage 2006 ; 273 : 4-11.

Bro E. Impact des cultures énergétiques sur la biodiversité. Synthèse bibliographique. CNERA PFSP, 2007.

Broyer I. Observatoire national de l'écosystème de prairie de fauche. Guide prairial. Chambre d'agriculture du Maine et Loire, 2006.

Bro $\mathrm{E}$, et al. Conservation de la Perdrix grise : la France au premier rang - bilan des 10 dernières années de suivi des populations. Faune Sauvage 2006 ; 272 : 22-30.

Bro $\mathrm{E}$, et al. La faune sauvage en milieux cultivés. Comment gérer le petit gibier et ses habitats. Plaquette ONCFS, 2007 : 79 p.

Chambre d'agriculture Pays-de-la-Loire ICTF. Couvrir les sols en automne. Guide pratique pour réussir sa culture intermédiaire. 2003.

Clergue B. Évaluation de l'impact des pratiques agricoles sur le fonctionnement de la biodiversité à l'aide d'indicateurs agri-environnementaux. Thèse : Sciences agronomiques. Nancy Université INPL, 2008 : 175 p.

Collectif. Les cultures ligno-cellulosiques et herbacées pour la production de biomasse à usage non alimentaire, fiche TCR de Peuplier. ADEME Éditions, 1998.

Collectif. Impact des TTCR de saule sur le paysage et la biodiversité. AILE, 2008.

Collectif. Créer une mare/dossier technique de la gazette des terriers. Documentation des clubs CPN, 1998 : 67 p.

Collectif. Gérer une mare/dossier technique de la gazette des terriers. Documentation des clubs CPN, 1999 : 74 p. 
Collectif. Productions végétales, pratiques agricoles et faune sauvage. UIPP, ACTA, ONCFS, 2007.

Collectif. Gestions de territoire, le cahier conseil. Chambre régionale d'agriculture de Picardie. 7, UIPP, ACTA, ONCFS, 2004.

Collectif. Les mouillères, les platières et les mares de l'öle-de-France. Bulletin de l'Association des naturalistes de la Vallée du Loing $2005 ; 81\left(\mathrm{n}^{\circ} 1\right): 60 \mathrm{p}$.

Collectif. Gestion des bords de champs cultivés: agriculture, environnement, faune sauvage. Éditions ONCFS, ATB, FNC, Preolia Cetiom, Arvalis, Syngenta, 2009.

Collectif. Planter des haies champètres. Un geste écocitoyen, connaître pour agir. Association régionale de l'environnement de HauteNormandie, 2000.

Collectif. Guide des bonnes pratiques pour l'entretien et la conception des fossés municipaux. Association pour la protection de I'environnement du Lac Saint-Charles et des Marais du Nord (APPEL), 2008.

Collectif. Une technique pour l'entretien des fossés. Ministère de l'Agriculture, Pêcheries et Alimentation Québec (MAPAQ), 2008.

Collectif. Les fossés/fiches documentaires. CREN Languedoc-Roussillon, 2007.

Collectif. Les anciens bras fl uviaux, iônes, boires, noues... CREN de Rhône-Alpes, 2007.

Collectif. Dossier spécial colloque national " agriculteurs, chasseurs: gérons ensemble la Perdrix grise ". Faune Sauvage 2008 ; 286 : 16-49.

Capitaine $\mathrm{M}$, et al. Gérer une mare: ce qu'il faut savoir pour entretenir et gérer une mare. Fed. des clubs connaître et protéger la nature (CPN), $1999: 74$ p.

Collectif. Étude d'impact sur l'environnement-Contournement de l'agglomération de Sherbrooke-Annexe D. Ministère des Transports du Québec, 2009.

Collectif. Le Haut-jet, taille de formation et élagage/Bocage info $n^{\circ} 1$. Mission Bocage, 2000.

Collectif. Guide technique de l'aménagement des bâtiments publics et ouvrages d'art des Deux-Sèvres en faveur des Chauves-Souris. Deux-Sèvre Nature environnement, 2006.

Collectif. Guide pour I'aménagement des combles et clochers des églises et d'autres bâtiments. Groupement nature de I'Institut royal des sciences naturelles de Belgique, 2003.

Collectif. Oiseaux et patrimoine bâti. Vous souhaitez construire ou rénover? Pensez aux oiseaux! LPO Vienne, 2006.

Collectif. Chouette Chevêche, Cahier technique. LPO France, 2006.
Collectif. Fiches techniques, aménagements chauves souris. Groupe mammologique Breton, 2005.

Collectif. Implantation de couverts végétaux en interculture. Cahier technique de I'Institut technique de la betterave, 2003.

Conservatoire - Études des écosystèmes de Provence (CEEP). Fiche inventaire des mares de la région PACA. 2003.

Conservatoire des espaces et paysages $\mathrm{d}^{\prime}$ Auvergne (CEPA). Fiche recensement des mares et mouilleres d'Auvergne.

Conservatoire des sites naturels de Picardie. Picardie buissonnière à la découverte du patrimoine naturel, 2002 : $37 \mathrm{p}$.

Conservatoire des sites naturels de Picardie. Picardie buissoniere à la découverte du patrimoine naturel, $2002: 37$ p.

Conservatoire fédératif des espaces naturels de Basse-Normandie (CFEN). Fiche inventaire mares.

Conservatoire régional d'espaces naturels de Poitou-Charentes. Fiche diagnostic CAD mares. Site Natura, 2000 ; Chaumes-d'Avon, 2005.

Conservatoire des sites naturels bourguignons. Réseaux mares de Bourgogne, plaquette les mares en Bourgogne, des zones humides à préserver.

CPN. Dossier de la Gazette des Terriers, le journal des clubs CPN. Tout ce qu'il faut savoir pour créer une mare.

CORPEN. Les fonctions environnementales des zones tampons - les bases scientifiques et techniques des fonctions de protections des eaux, 2007 : 75 p.

Coufourier N. Un manteau d'hiver régénérant. Réussir céréales grandes cultures, $2008 ; n^{\circ} 214$.

Coulon F, Pointereau P, Meiffren I. Le préverger pour une agriculture durable - Guide technique. Éditions SOLAGRO, 2005.

CPIE Woëvre-Côtes-de-Meuse, Conseil régional de Lorraine. Recommandation du bon entretien des ruisseaux à l'usage des agriculteurs. Agence régionale de l'environnement en Lorraine, 2001.

Decourtye A, Lecompte P, Pierre J, Chauzat MP, Thiébeau P. Introduction de jachères florales en zones de grandes cultures - Ou comment mieux concilier agriculture et biodiversité, et par conséquent, l'apiculture. Le courrier de l'environnement de I'INRA 2007.

Decourtye $A$, Tisseur $M$. Jachères florales Bonnes pour les abeilles donc bonnes pour l'agriculture. Perspectives agricoles 2007.

Di-cintio F, Meurin E. Mise en place et suivi de parcelles expérimentales de saules dans le Pays $d^{\prime}$ Epinal. Chambre d'agriculture des Vosges, 2008.
DIREN Centre et conservatoire botanique national Bassin Parisien. Guide des habitats naturels déterminants ZNIEFF de la région Centre.

Dijkstra KDB, Lewington R. Guide des libellules de France et d'Europe. Paris: Delachaux Niestle, 2007 : 320 p.

Diwo Allain S, Rougon D, Lemesle B, Viaux P. Fiche "Carabes: auxiliaires des cultures et indicateurs de la biodiversité d'un milieu", CRITT Innophyt, 2003.

Dommanget JL. Les libellules et leurs habitats. Caractéristiques générales. Éléments de gestion et de restaurations. Bois d'Arcy: Soc. Fr. Odonatologie, 1998 : $20 \mathrm{p}$.

Dupraz C, Liagre F. Agroforesterie - Des arbres et des cultures. Éditions Agricole, 2008. ISBN : 978-2-85557-150-8.

Dupraz C, Liagre F. Agroforesterie: des arbres et des cultures. Éditions France Agricole, 2008.

Bruère A. Pour une réhabilitation de la flore messicole en Meuse. Mise en évidence des facteurs favorisant son maintien. Mémoire de fin d'études, 2005.

Fairon J, et al. Guide pour l'aménagement des combles et clochers des églises et d'autres bâtiments. Brochure technique $n^{\circ} 4$. Institut royal des sciences naturelles de Belgique. Groupement Nature, 2003 : 79 p.

Favre S. Analyser les résultats de 79 diagnostics d'exploitation pour en tirer des enseignements en terme de méthode de conseil sur la biodiversité, rapport de fin d'etudes ingénieur de I'ENSAT, 2010.

Fort D. L'agroforesterie: des arbres au coeur des champs. Forêt magazine, 2004.

François R. Note sur la distribution et l'écologie du Lézard vert (Lacerta viridis) dans I'Oise. PIC MAR, $1998 ; n^{\circ} 4: 28-31$.

FRC Franche-Comté. Catalogue technique pour la conception et la valorisation des haies champêtres en Franche-Comité. 2008.

Joannon, Bro, Thenail, Baudry. Crop patterns and habitat préferences of the grey partridges farmaland bird in agronomy for sustainable development. 2008.

Judeaux S. L'entretien des bords de champs. Bulletin de la FRCUMA Ouest, 2005.

Laffitte et al. Guide technique de la mare en Caps et Marais d'Opale. Parc naturel régional des Caps et Marais d'Opale, 2005 : 36 p.

Liagre F. Les Haies rurales: rôles, création, entretien. Paris: Éditions France Agricole, 2006.

Gadoum S, Terzo M, Rasmont P. Jachères apicoles et jachères fleuries: la biodiversité au menu de quelles abeilles? Courrier de I'environnement de I'INRA $n^{\circ} 54$, septembre $2007:$ p 57. 
Gloria C. Un manteau d'hiver régénérant. Réussir céréales grandes cultures, 2008 : $\mathrm{n}^{\circ} 214$.

Grand D, Boudot JP. Les libellules de France, Belgique et Luxembourg. Collection Parthénope, 2007.

Hani F, Popow G, Reinhard H, Shawarz A, Tanner K. Protection des plantes en production intégrée, Grandes Cultures. Édition LmZ, 2004.

Havet P. Gestion de l'espace, pratiques phytosanitaires et faune sauvage - Eléments d'analyse et conseils de base. Phytoma, 2000.

ITAB. Maîtriser les adventices en grandes cultures biologiques. Guide technique, 2005.

Chambre régionale d'agriculture de Picardie. Le cahier conseil "Gestion de territoire", 2004.

Labreuche J. Cultures intermédiaires: enjeux et mise en œuvre. Arvalis, 2007.

Landmann G, Gosselin F, Bonhême I, (Eds). Bio2, Biomasse et Biodiversité forestieres. Augmentation de l'utilisation de la biomasse forestiere: implications pour la biodiversité et les ressources naturelles. Paris: MEEDDM-ecoflor, 2009.

Larrieu L, Gonin P. L'Indice de biodiversité potentielle (IBP): une méthode simple et rapide pour évaluer la biodiversité potentielle des peuplements forestiers. Revue Forestiere Française 2009 ; 6-2008 : 727-48.

Le Roux X, Barbault R, Baudry J, et al. (Eds.). Agriculture et biodiversité. Valoriser les synergies. Expertise scientifique collective, rapport, INRA (France), 2008.

Liagre F. Enjeux de l'agroforesterie. Alter Agri $2008 ; n^{\circ} 91: 11-3$.

Liagre F. L'agroforesterie en France. www. wervel.be/downloads/FabienLiagre-frans.

ppt, 2006.

Masson B. Agroforesterie: quand écologie rime avec rentabilité. Agra Valor 2006; $\mathrm{n}^{\circ} 141: 6-9$.

Mayot P. Lettre du développement. Chambre d'agriculture de la Sarthe, 2007.

Maillet-Mezeray J. Bandes enherbées enjeux, implantation et entretien. Éditions Arvalis, 2007: 40 p.

Maillet-Mezeray J. Diagnostics des bords de champs, mode d'emploi et propositions. Perspectives Agricoles 2007 ; 334 ; 6-9.

Messean A. Étude des prairies du bassin versant du Goujon. Conservatoire des sites naturels de Picardie, 2009 : 10 p.

Mestelan P, de Sainte Marie C, Vansteelant JIY. Guide pour la mise en œuvre de l'engagement unitaire agro-environnemental " main/en de la richesse floristique d'une prairie naturelle» (Herbe_07). INRA Avignon, 2008.
Naulleau G. Les lézards de France. Revue française $d^{\prime}$ aquariophilie, $17^{\mathrm{e}}$ année, $\mathrm{n}^{\circ} 3$ et 4. Université de Nancy, $1990: 128$ p.

Noblet JF. La nature sous son toit: hommes et bêtes: comment cohabiter? Éditions Delachaux et Niestlé, 2005.

Ouvrard N. Les cultures intermédiaires pour limiter l'érosion. Réussir céréales grandes cultures, $2008: \mathrm{n}^{\circ} 214$.

Omnes G. Agroforesterie : un capital sur pied dans ses parcelle de céréales. La France Agricole, $2005: 32$.

Petit et al. Agriculture, prairies de fauche et environnement dans le Massif Jurassien. Outil de diagnostic et conseil. Guide Technique 2005. GIS Alpes du Nord. Parc Naturel Régional Haut-Jura, 2005 : 49 p.

Pierroux A. Risques infectieux et parasitaires liés à l'abreuvement dans les mares. Mémoire bibliographique, Conservatoire fédératif des espaces naturels de Basse-Normandie (Mondeville), 2008 : 26 p.

Pointereau et al. Les impacts environnementaux et paysagers des nouvelles productions énergétiques sur les parcelles et bâtiments agricoles. 2009.

Regnault-Roger C, et al. Enjeux phytosanitaires pour l'agriculture et l'environnement. Ed. Tec et Doc, 2005.

Regnault Roger C, Philogene BJR. Biopesticides d'origine végétale. Éditions Tec et Doc. Lavoisier, 2002.

Riegel I, Soufflot I. Protéger I'Effraie des clochers, cahier technique. LPO France, 2007.

Sajaloli B, Limoges O, Dutilleul C, Thulie A. Contribution des mares à la qualité biologique et sociale des territoires/exemples dans le Bassin Parisien. Colloque "l'eau de la cellule au paysage ». Elsevier, $2000:$ 215-34.

Soltner D. Bandes enherbées et autres dispositifs bocagers/Guide Agriculture et biodiversité "Aménagements et pratiques favorables à la biodiversité ». 2001.

Soltner D. Les techniques culturales simplifiées, tome 2 ; les TCS, comment? Sciences et techniques agricoles, 2000.

Thomas F. Les mélanges de couverts végétaux. Techniques Culturales Simplifiées, 2005.

Trotignon J. Des étangs pour la vie/améliorer la gestion des étangs. Cahiers Techniques $2000 ; n^{\circ} 61: 70$ p.

Vallance M. Faune sauvage de France - biologie, habitats et gestion. Éditions du GerfautONCFS, $2007: 415$ p.

Viaux P. Une troisieme voie en Grande Culture. Éditions Agridécisions, 1999.

Vilain Lionel. La méthode IDEA. Educagri éditions, $2008: 184 \mathrm{p}$.
Wartelle R. (dir.). Gestion de territoire, le cahier conseil. Chambre régionale d'agriculture de Picardie, 2004.

Zucchi H. La prairie, un environnement menacé. Découverte de la nature. Ulissedi/ ons, 1989 : 127 p.Site Web

www.mares-franche-comte.org

www.les-mares.com

http://www.oncfs.gouv.fr

http://www2.mnhn.fr/vigie-nature/

http://www.rspb.org.uk

http://www.oiseaux.net/oiseaux/vanneau. huppe.html

Inventaire des mares d'Alsace : http://observatoirealsace.free.fr/plume/mares

http://cheveche.Ipo.fr

Arbre \& Paysage 32 : http://www.arbre-etpaysage32.com

http://www.rhone-alpes.chambagri.fr/phytov3/ pages/haies.htm

Univers Nature : http://www.univers-nature. com/dossiers/haie.html

http://www.lesbocages.be

http://www.haiesvives.org/PDF/Droits_arbre. pdf.

http://www.syndicat-vistre.fr/Agricole/fosses. php

http://www.rappel.qc.ca/des-fosses-ecologiques-et-economiques.html

http://www.cemagref.fr/INformations/DossiersThematiques/EauAgriculture/Recherche04.htm http://www.gesteau.eaufrance.fr/

http://www.refugespourleschauves-souris.com/ http://www.lpo.fr/refugeslpo/conseils/fiches/ index.shtml

http://dsne.chez-alice.fr/chiro/

http://www.observatoire-environnement.org/ dsne/IMG/pdf/guide-technique-web.pdf

www2.ademe.fr/servlet/getDoc?cid=96\&m= $3 \& \mathrm{id}=51256 \& \mathrm{p} 1=00 \& \mathrm{p} 2=11 \& \mathrm{ref}=17597$

www.agrilianet.com/pdf/brochure_03_couvrir_ les sols.pdf

http://www.gissol.fr/actualite/Documents/ Guide-BV.pdf

Descriptif des habitats Natura 2000 liés aux prairies : http://natura2000.environnement. gouv.fr/habitats/cahiers.html

ACTA ; Logiciel PhytActa 2008 : http://www. acta.asso.fr/

FN CUMA : qualité de l'eau et réduction des transferts de produits phytosanitaires : http:// www.ouest.cuma.fr/Documents/machinismeenvironnement/pulverisation-et-desherbage-mecanique/Technique-alternative-dedesherbage/Generalites/plaquette-klit-eauet-reduction-produits-phytos.pdf 
INRA : des itinéraires techniques à bas niveaux d'intrants pour des variétés rustiques de blé tendre: une alternative pour concilier économie et environnement : http://www.inra.fr/dpenv/pdf/rollac49.pdf

INRA, 2010, Ecophyto R\&D Quelles voies pour réduire I'usage des pesticides ? Synthèse du rapport d'étude, janvier 2010, 90p

INRA, 2008, Agriculture et biodiversité. Valoriser les synergies. Expertise scientifique collective : http://www.inra.fr/I_institut/ expertise/expertises_realisees/agriculture_et_ biodiversite_rapport_d_expertise

INRA, Cemagref, 2005, Expertise scientifique collective, pesticides agriculture et environ- nement, réduire l'utilisation des pesticides et en limiter les impacts environnementaux : http://www.inra.fr/I_institut/missions_et_ strategie/les_missions_de_I_inra/eclairer_ les_decisions/pesticides_rapport_d_expertise ONCFS ; outil "Choisissez et Dosez": http://www.oncfs.gouv.fr

Association française d'agroforesterie : http:// www.agroforesterie.fr

Association française de l'arbre et de la haie champêtre : http://www.afahc.fr

Agroof développement :http://www.agroof. net

Le DVD «Agroforesterie - Enjeux et perspectives " est également visible sur le site internet : http://www.agroof.net/agroof_ edition/agroof_DVD_13.html

Phyt'Auvergne : étude bibliographique des solutions techniques et agronomiques permettant de réduire l'usage de produits phytosanitaires en grandes cultures : http:// www.phyteauvergne.ecologie.gouv.fr/IMG/ html/Techniques_alt_agro/Etude_biblio.htm http://www.foretpriveefrancaise.com/indicede-biodiversite-potentielle-ibp-696129.html

Site du GIP ECOFOR (ECOsystèmes FORestiers) : ECOFOR : http://www.gip-ecofor.org/ publi/page. php? $\mathrm{id}=45 \& \mathrm{rang}=0 \&$ domain $=$ $1 \&$ lang=fr_FR 